\title{
New-onset psychogenic non-epileptic seizures in a patient with epilepsy
}

\section{The importance of inpatient monitoring on an epilepsy unit to ensure appropriate diagnosis and treatment}

Kendra L Derry (Meds 2015)

Faculty Reviewers: Dr Paul A Derry, PhD (C Psych) (Department of Psychology) \&

Dr Jorge G Burneo, MSPH, MD (Department of Clinical Neurological Sciences)

\section{INTRODUCTION}

Epileptic seizures are paroxysmal behavior patterns due to central nervous system (CNS) dysfunction. ${ }^{1}$ Psychogenic non-epileptic seizures (PNES) are disorders that can be mistaken for epilepsy. However, unlike genuine epilepsy, PNES are not the consequence of abnormal electrical discharges in the brain. The Diagnostic and Statistical Manual of Mental Disorders, Fourth Edition (DSM-IV) categorizes PNES under Somatoform Disorders: Conversion Disorder along with seizures or convulsions. ${ }^{2}$ PNES involves unconscious physical manifestation of psychosocial distress.

As PNES and epileptic seizures share many features, it can be difficult to differentiate between the two. ${ }^{3}$ Diagnosis of PNES is further complicated in cases where there is a concomitant diagnosis of epilepsy. Data suggests that the prevalence of co-existing epilepsy with PNES lies between 10 and $50 \% .^{4-7}$

It is important to accurately diagnose PNES because treatment regimens that are appropriate for epileptic seizures (medication and possible epilepsy surgery) are inappropriate, ineffectual, costly and potentially harmful in the treatment of PNES.

We present a case of new onset PNES in a patient with previously diagnosed epilepsy. This case exemplifies how an accurate diagnosis, achieved through admission to the Epilepsy Monitoring Unit (EMU), can drastically alter prognosis and management. This case also highlights the importance of a multidisciplinary approach when dealing with conversion disorders.

\section{CASE PRESENTATION}

Ms X is a 44-year-old married woman who was admitted to the University Hospital EMU in August 2013, for re-investigation as a surgical candidate for her previously diagnosed epilepsy.

In March of 2004 Ms X experienced her first seizure. On November 7, $2006 \mathrm{Ms}$ X was admitted to the EMU for investigation. Subdural electrodes were implanted and localized the origins of the seizures to the left anterior temporal and left orbitofrontal region. A diagnosis of left fronto-temporal epilepsy was made. At this time Ms X was offered a left temporal lobectomy plus posterior inferior frontal lobectomy. The subdural electrodes were explanted on November 30, 2006 with no complications, and the patient was discharged home December 1, 2006 with full medications. At the time, Ms X wished to proceed with surgery and was to be scheduled for the procedure. On December 14, 2006 Ms X presented to the Emergency Room in London with a headache. It was discovered that she had developed bilateral temporal lobe abscesses. Incision and needle drainage of the left postero-temporal brain abscess was performed and ceftriaxone was administered for 6 weeks. Ms X recovered well from the infection and it was the surgeon's opinion that her infection would not interfere with her ability to undergo surgery. However, the patient decided to defer surgery and opted for medical management.

With continued problematic seizures, Ms X recently decided she wished to pursue surgery. As is standard practice, she was re-admitted for further seizure monitoring to confirm seizure origin. In contrast to the seizure activity recorded in 2006, all events recorded during her 2013 evaluation revealed entirely normal EEGs. No epileptic seizures were recorded. Ms X did however, experience numerous PNES which were recorded via continuous EEG/video monitoring. In contrast to her seizures in 2006, her current spells are long in duration ( 5 to $45 \mathrm{~min}$ ) and non-stereotyped, with asynchronous thrashing of arms and legs and retained consciousness. The patient reported she experiences about one event per day.

Ms X's past medical history is significant for migraines (once per week), anxiety disorder with panic attacks and an anoxthalmic left eye.

Medications at the time included

1. Atenolol $50 \mathrm{mg}$

2. Vimpat $200 \mathrm{mg}$ twice daily

3. Topamax $100 \mathrm{mg}$ twice daily

4. Dilantin 350mg once at nighttime

Ms X has a significant psychological history. As a teenager she was in a physically abusive relationship and she was sexually assaulted. In early adulthood, her first husband and her father both unexpectedly passed away. Three years ago she suffered multiple leg fractures in a serious snowmobile accident, and underwent several surgeries and significant rehabilitation.

On admission, Ms X's physical examination, including a detailed neurologic exam, was normal.

Psychological assessment included formal psychological testing, which indicated the diagnosis of conversion disorder. 


\section{DISCUSSION}

The absence of electrographic evidence of epileptic seizures during Ms X's most recent admission to the EMU was an unanticipated finding. In 2006, her EEG recordings showed clear evidence of left fronto-temporal epilepsy. No PNESs were recorded at that time. Over the nine years since her diagnosis, it was presumed her epilepsy was refractory to medical therapy, as her seizures were uncontrolled by medications alone. Epilepsy surgery seemed to be the most appropriate treatment option for this patient. The importance of re-investigating her is demonstrated by the realization that her epileptic seizures are now apparently well-controlled, and that her problem is caused by new symptoms that are non-epileptic in origin. Discovering this prevented brain surgery and allowed for implementation of appropriate treatment.

The diagnosis of PNES can be difficult. Intensive video-EEG monitoring in the EMU remains the gold standard for the diagnostic evaluation of patients with conversion disorder. ${ }^{8}$ Video-EEG simultaneously records a patient's behavior and brain electrical activity. ${ }^{9}$ When video-EEG monitoring captures a characteristic spell while the EEG background is normal before, during and after the event, a diagnosis of PNES should be considered.

Although there are no pathognomonic clinical signs that distinguish PNES from epileptic seizures, there are some risk factors and warning signs. Psychogenic seizures are characteristically longer in duration, ${ }^{10}$ with motor manifestations that tend to involve non-stereotyped, asynchronous, non-rhythmical thrashing of the extremities. ${ }^{11}$ This is in comparison to epileptic seizures which (1) tend to onset earlier in life, (2) are usually brief and stereotyped and (3) generally respond at least somewhat to medication. Additionally, retained consciousness and retrospective recall of ictal events is more indicative of PNES.

Psychiatric comorbidities are also common in patients with PNES. The most common include depression, anxiety, ${ }^{12}$ post-traumatic stress disorder and personality disorders. ${ }^{13}$ A history of physical abuse is more likely in PNES ( $75 \%)$ than in epilepsy $(\sim 42 \%)$. Further, the rate of sexual abuse is higher in the PNES population $(\sim 42 \%)$ compared to the epilepsy population (19\%). ${ }^{10}$

Ms X has a good prognosis. She accepts that her current spells are psychogenic in origin and she is attending counseling. Since no epileptic seizures were recorded during her admission, we assume that her epilepsy is well controlled with medications. It is not uncommon for epileptic medications to be increased and/or added to the regime to attempt to control seizures in cases similar to this one. Inappropriate treatment, however, exposes the patient to the adverse effects of antiepileptic drugs and socioeconomic hardships - patients may be unable to work or drive or have limited social activities - and adds to the burden on healthcare resources.

In summary, this case highlights the difficulty in distinguishing between epilepsy and PNES, and emphasizes the importance of both neurological and psychological evaluation for developing a correct diagnosis. It is important to appreciate that some patients with PNES also have genuine epileptic attacks that do require anticonvulsant medications.

\section{REFERENCES}

1. Leppik I. Contemporary diagnosis and management of the patient with epilepsy. 3rd ed. Newtown (PA): Handbooks in Health Care; 1997.

2. Michael B, editor. Diagnostic and statistical manual of mental disorders. 4th ed. [Internet]. Washington, DC: American Psychiatric Association; 2000 [cited 2013 Aug 22]. Available from http://online.statref.com/Document.aspx?FxId=37\&SessionId=1BC2793WJJQFNMKD\#H\&0\&Chapters Tab\&APFTKtW5n3gfGFj5b7FkLg\%3d\%3d\&\&37

3. Lesser RP. Psychogenic seizures. Neurology. 1996 Jun;46(6):1499-507.

4. Benbadis SR, Allen Hauser W. An estimate of the prevalence of psychogenic non-epileptic seizures. Seizure. 2000 Jun;9(4):280-1.

5. Benbadis SR, Agrawal V, Tatum WO. How many patients with psychogenic nonepileptic seizures also have epilepsy? Neurology. 2001 Sep;57(5):915-7.

6. Reuber M, Elger CE. Psychogenic nonepileptic seizures: review and update. Epilepsy Behav. 2003 Jun;4(3):205-16.

7. O’Sullivan SS, Spillane JE, McMahon EM, Sweeney BJ, Galvin RJ, McNamara B, Cassidy EM. Clinical characteristics and outcome of patients diagnosed with psychogenic nonepileptic seizures: A 5-year review. Epilepsy \& Behaviour. 2007 Aug;11(1):77-84.

8. Krumholz A. Nonepileptic seizures: diagnosis and management. Neurology. 1999;53(5 Suppl 2):S76-S83.

9. Alsaadi TM, Thieman C, Shatzel A, Farias S. Video-EEG telemetry can be a crucial tool for neurologists experienced in epilepsy when diagnosing seizure disorders. Seizure.2004 Jan;13(1):32-4.

10. Cragar DE, Berry D, Fakhoury TA, Cibula JE, Schmitt F. A review of diagnostic techniques in the differential diagnosis of epileptic and nonepileptic seizures. Neuropsychol Rev. 2002 Mar;12(1):31-64.

11. Boon PA, Williamson PD. The diagnosis of pseudoseizures. Clin Neurol Neurosurg. 1993 Mar;95(1):1-8.

12. Tellez Zenteno JF, Patten SB, Jetté N, Williams J, Wiebe S. Psychiatric comorbidity in epilepsy: a population based analysis. Epilepsia. 2007 Dec;48(12): 2336-44.

13. Bowman, ES. Nonepileptic seizures: psychiatric framework, treatment, and outcome. Neurology.1999;53(5 Suppl 2):S84-8. 